\title{
Robot-Assisted Upper Limb Training for Patients with Multiple Sclerosis: An Evidence-Based Review of Clinical Applications and Effectiveness
}

\author{
Sofia Straudi ${ }^{1}$, Marco Tramontano ${ }^{2}\left(\mathbb{D}\right.$, Emanuele Francesco Russo ${ }^{3}$, Luca Perrero ${ }^{4}$, Michela Agostini ${ }^{5}{ }^{\circ}$, \\ Marialuisa Gandolfi $\left.{ }^{6}{ }^{(}\right)$, Irene Aprile ${ }^{7, *}$, Matteo Paci ${ }^{8}{ }^{(0)}$, Emanuela Casanova ${ }^{9}$, Dario Marino ${ }^{10}$, \\ Giuseppe La Rosa ${ }^{11}{ }^{10}$, Federica Bressi ${ }^{12}$, Silvia Sterzi ${ }^{12}$, Daniele Giansanti ${ }^{13}$, Alberto Battistini ${ }^{9}$, \\ Sandra Miccinilli ${ }^{12}$, Serena Filoni ${ }^{3}$, Monica Sicari ${ }^{14}$, Salvatore Petrozzino ${ }^{14}$, Claudio Marcello Solaro ${ }^{15}(\mathbb{D}$, \\ Stefano Gargano ${ }^{16}$, Paolo Benanti ${ }^{17}{ }^{1}$, Paolo Boldrini ${ }^{18}$, Donatella Bonaiuti ${ }^{18}$, Enrico Castelli ${ }^{19}$, \\ Francesco Draicchio ${ }^{20}$, Vincenzo Falabella ${ }^{21}$, Silvia Galeri ${ }^{16}$, Francesca Gimigliano ${ }^{22}$, Mauro Grigioni ${ }^{13}{ }^{(0)}$, \\ Stefano Mazzoleni ${ }^{23}$, Stefano Mazzon ${ }^{24}$, Franco Molteni ${ }^{25}$, Maurizio Petrarca ${ }^{26}$, Alessandro Picelli ${ }^{6}{ }^{\circ}$, \\ Federico Posteraro ${ }^{27}$, Michele Senatore ${ }^{28}$, Giuseppe Turchetti ${ }^{29} \mathbb{( D}$, Giovanni Morone ${ }^{2} \mathbb{D}$ and \\ Working Group Upper Limb “CICERONE” Italian Consensus Conference on Robotic Rehabilitation †
}

check for updates

Citation: Straudi, S.; Tramontano, M.; Russo, E.F.; Perrero, L.; Agostini, M.; Gandolfi, M.; Aprile, I.; Paci, M.;

Casanova, E.; Marino, D.; et al.

Robot-Assisted Upper Limb Training for Patients with Multiple Sclerosis: An Evidence-Based Review of

Clinical Applications and

Effectiveness. Appl. Sci. 2022, 12, 222.

https: / / doi.org/10.3390/

app12010222

Academic Editors: Claudio Belvedere and Redha Taiar

Received: 21 September 2021

Accepted: 20 December 2021

Published: 27 December 2021

Publisher's Note: MDPI stays neutral with regard to jurisdictional claims in published maps and institutional affiliations.

Copyright: (C) 2022 by the authors. Licensee MDPI, Basel, Switzerland. This article is an open access article distributed under the terms and conditions of the Creative Commons Attribution (CC BY) license (https:// creativecommons.org/licenses/by/ $4.0 /)$.
1 Neuroscience and Rehabilitation Department, Ferrara University Hospital, 44122 Ferrara, Italy; sofia.straudi@gmail.com

2 Santa Lucia Foundation, IRCCS, 00179 Rome, Italy; m.tramontano@hsantalucia.it (M.T.); g.morone@hsantalucia.it (G.M.)

3 Padre Pio Foundation and Rehabilitation Centers, 71013 San Giovanni Rotondo, Italy; emanuele.f88@gmail.com (E.F.R.); serena.diba@gmail.com (S.F.)

4 Neurorehabilitation Unit, Azienda Ospedaliera SS. Antonio e Biagio e Cesare Arrigo, 15121 Alessandria, Italy lperrero@ospedale.al.it

5 Department of Neuroscience, Section of Rehabilitation, University-General Hospital of Padova, 35122 Padova, Italy; michela.agostini@unipd.it

6 Department of Neurosciences, Biomedicine and Movement Sciences, University of Verona, 37134 Verona, Italy; marialuisa.gandolfi@univr.it (M.G.); alessandro.picelli@univr.it (A.P.)

7 IRCCS Fondazione Don Carlo Gnocchi ONLUS, 50143 Florence, Italy

8 AUSL (Unique Sanitary Local Company) District of Central Tuscany, 50122 Florence, Italy; matteo.paci@applicazione.it

9 IRCCS Istituto delle Scienze Neurologiche di Bologna, UOC Medicina Riabilitativa e Neuroriabilitazione, 40139 Bologna, Italy; e.casanova@ausl.bologna.it (E.C.); alberto.battistini@fastweb.net (A.B.)

10 IRCCS Neurolysis Center "Bonino Pulejo", 98124 Messina, Italy; dario.marino95@gmail.com

11 C.S.R. Consorzio Siciliano di Riabilitazione, 95123 Catania, Italy; laros.giu@gmail.com

12 Physical Medicine and Rehabilitation Unit, Campus Bio-Medico University, 00128 Rome, Italy; f.bressi@unicampus.it (F.B.); s.sterzi@unicampus.it (S.S.); s.miccinilli@unicampus.it (S.M.)

13 National Center for Innovative Technologies in Public Health, Italian National Institute of Health, 00161 Rome, Italy; daniele.giansanti@iss.it (D.G.); mauro.grigioni@iss.it (M.G.)

14 A.O.U. Città della Salute e della Scienza di Torino, 10126 Torino, Italy; monicasicari80@gmail.com (M.S.); salvatore.petrozzino@gmail.com (S.P.)

15 CRRF “Mons. Luigi Novarese”, 13040 Moncrivello, Italy; csolaro@libero.it

16 IRCCS Fondazione Don Carlo Gnocchi ONLUS, 20148 Milan, Italy; stgargano@dongnocchi.it (S.G.); sgaleri@dongnocchi.it (S.G.)

17 Faculty of Theology, Pontifical Gregorian University, 00187 Rome, Italy; benanti@unigre.it

18 Società Italiana di Medicina Fisica e Riabilitativa (SIMFER), 00198 Roma, Italy; paolobold@gmail.com (P.B.); dbonaiuti2@yahoo.it (D.B.)

19 Paediatric Neurorehabilitation, IRCCS Bambino Gesù Children's Hospital, 00165 Rome, Italy; enrico.castelli@opbg.net

20 Department of Occupational and Environmental Medicine, Epidemiology and Hygiene, INAIL, Monte Porzio Catone, 00185 Rome, Italy; f.draicchio@inail.it

21 President Italian Federation of Persons with Spinal Cord Injuries (Faip Onlus), 00195 Rome, Italy; falabella@fishonlus.it

22 Department of Mental and Physical Health and Preventive Medicine, University of Campania "Luigi Vanvitelli", 80138 Naples, Italy; francescagimigliano@gmail.com

23 Department of Electrical and Information Engineering, Politecnico di Bari, 70125 Bari, Italy stefano.mazzoleni@poliba.it

24 AULSS6 (Unique Sanitary Local Company) Euganea Padova, 35131 Padova, Italy; stefano.mazzon@gmail.com 
25 Villa Beretta Rehabilitation Center, Department of Rehabilitation Medicine, Valduce Hospital, 23845 Costa Masnaga, Italy; fmolteni@valduce.it

26 Movement Analysis and Robotics Laboratory MARlab, IRCCS Bambino Gesù Children's Hospital, 00163 Rome, Italy; maurizio.petrarca@opbg.it

27 Versilia Hospital AUSL Toscana Nord Ovest, 55049 Lucca, Italy; federico.posteraro@uslnordovest.toscana.it

28 Past President A.I.T.O.-Associazione italiana terapisti occupazionali, 00136 Rome, Italy; presidente@aito.it

29 Management Institute, Sant'Anna School of Advanced Studies, 56127 Pisa, Italy; giuseppe.turchetti@santannapisa.it

* Correspondence: iaprile@dongnocchi.it; Tel.: +39-0633086545

+ Collaborators: Angela Palomba (Department of Medical and Surgical Specialties and Dentistry, University of Campania "Luigi Vanvitelli", Naples, Italy), Chiara Arienti (IRCCS Fondazione Don Carlo Gnocchi ONLUS, Milan, Italy; carienti@dongnocchi.it), Ludovica Baluardo (Ferrara University Hospital, Neuroscience and Rehabilitation Department, Ferrara, Italy), Alex Martino Cinnera (Santa Lucia Foundation, IRCCS, Rome, Italy; a.martino@hsantalucia.it), Manuela Desilvestri (Neurorehabilitation Unit, Azienda Ospedaliera SS. Antonio e Biagio e Cesare Arrigo, Alessandria, Italy, EU; mdesilvestri@ospedale.al.it), Marco Bravi (Campus Bio-Medico University Hospital, Italy; m.bravi@unicampus.it), Diletta Bruno (Campus Bio-Medico University Hospital, Italy; d.bruno@unicampus.it), Fabio Santacaterina (Campus Bio-Medico University Hospital, Italy), Cristiano Pecchioli (Department of Neuroscience and Neurorehabilitation, IRCCS San Raffaele Pisana, 00167 Rome, Italy; f.santacaterina@unicampus.it), Valentina Boetto (Università degli Studi di Torino, Torino, Italy).

Abstract: Upper extremities limitation is a common functional impairment in patients with Multiple Sclerosis (PwMS). Novel technological devices are increasingly used in neurorehabilitation to support motor function improvement and the quantitative assessment of motor performance during training in patients with neurological diseases. In this review, we systematically report the evidence on clinical applications and robotic-assisted arm training (RAT) in functional recovery in PwMS. PubMed/MEDLINE, the Cochrane Library, and the Physiotherapy Evidence Database (PEDro) databases were systematically searched from inception to March 2021. The 10-item PEDro scale assessed the study quality for the RCT, and the AMSTAR-2 was used to assess the quality of the systematic review. The 5-item Oxford CEBM scale was used to rate the level of evidence. A total of 10 studies (161 subjects) were included. The selected studies included one systematic review, four RCTs, one randomized crossover, and four case series. The RCTs were scored as high-quality studies, while the systematic review was determined to be of low quality. Shoulder range of motion, handgrip strength, and proximal arm impairment improved after RAT. Manual dexterity, arm function, and use in daily life also ameliorated arm function. The high clinical heterogeneity of treatment programs and the variety of robot devices affects the generalizability of the study results; therefore, we emphasize the need to standardize the intervention type in future studies that evaluate the role of robotic-assisted training in PwMS. Robot-assisted treatment seems safe and useful to increase manual dexterity and the quality of movement execution in PwMS with moderate to severe disability. Additional studies with an adequate sample size and methodological rigour are warranted to drive definite conclusions.

Keywords: multiple sclerosis; robotic devices; rehabilitation; exoskeleton

\section{Introduction}

Multiple sclerosis (MS) is an inflammatory, neurodegenerative, demyelinating disorder of the Central Nervous System [1] with pathology progression that is highly variable among patients [2]. About 76\% of patients with MS (PwMS) experience upper limb dysfunction [3], and 30\% show functionally relevant cerebellar deficits such as tremor and ataxia [4,5]. Among them, 68\% show bilateral disorders in sensation, strength, and dexterity [6,7]. Moreover, robot-derived measures have revealed that PwMS show irregular arm trajectories; abnormalities in muscle synergies, timing, and organization [8]; and impaired movement execution, even at the early stages of the disease [9]. The clinical manifestations of MS include a wide range of neurological symptoms that can compromise essential functions, including cognitive and sensorimotor skills, resulting in deficits such as muscle weakness 
and lack of movement coordination. These limitations negatively influence the quality of life [10], home integration, and social participation [11,12] of PwMS.

While MS is degenerative, the impact of MS on disability progression can be limited by functional reorganization processes that leverage neural plasticity [13]. Indeed, data show that motor-learning skills [14] and force field adaptation during a robot-assisted reaching task [9] are still present in PwMS. This makes PwMS good candidates for rehabilitative strategies that build on plasticity and learning, such as arm robotics. These techniques provide a motivating, cognitive-engaged environment for high-intensity skilled movement training [15,16] and are thought to engage neuroplasticity [17] and learning [18] to mediate their effects. While robot-assisted rehabilitation was originated in the stroke field [19,20], in PwMS, it has been extensively studied for gait rehabilitation [21]. However, treatment efforts that are focused on upper-limb rehabilitation are especially necessary in the advanced stages of the disease, and studies show that multidisciplinary and robotassisted rehabilitation approaches are capable of improving the upper limb capacity in PwMS [22,23].

Considering the peculiar pathophysiology of disorders in Multiple Sclerosis, which differs from cerebrovascular pathophysiology, it is essential to identify specific fields for robotic device applications for PwMS. Therefore, this review aims to systematically report the evidence on the effectiveness of the clinical applications of robotic-assisted upper limb-therapy in PwMS.

\section{Materials and Methods}

The Upper Limb Robotics working group carried out the present study within the framework of the Italian Consensus Conference on "Rehabilitation assisted by robotic and electromechanical devices for persons with disability of neurological origin" (CICERONE), promoted by the Italian Society of Physical and Rehabilitation Medicine (SIMFER, Società Italiana di Medicina Fisica e Riabilitativa) and the Italian Society of Neurological Rehabilitation (SIRN, Società Italiana di Riabilitazione Neurologica) (2019-2021) [24].

\subsection{Search Strategy}

PubMed/MEDLINE, the Cochrane Library, and the Physiotherapy Evidence Database (PEDro) databases were systematically searched from their inception to March 2021 using the following keywords: "multiple sclerosis" and "robot" and "upper limb". An example of the full search strategy for PubMed/MEDLINE is as follows: (Multiple Sclerosis [Title/Abstract]) OR Multiple sclerosis [MeSH Terms] AND ((((robot*[Title/Abstract]) OR exoskeleton[Title/Abstract]) OR end-effector[Title/Abstract]) OR robotics[MeSH Terms]) OR Exoskeleton Device[MeSH Terms] AND ((((()upper limb[Title/Abstract]) OR upper extremity[Title/Abstract]) OR hand[Title/Abstract]) OR arm[Title/Abstract]) OR upper extremity[MeSH Terms])). This systematic review was performed in accordance with the Preferred Reporting Items for Systematic Reviews and Meta-analyses (PRISMA) statement [25].

\subsection{Selection Criteria}

After duplicates were removed, two reviewers independently screened for inclusion based on the title and abstract of all of the potentially relevant studies that had been identified. In case of disagreement, a consensus was achieved by the decision of a third reviewer. Full-text studies were subsequently retrieved by the same two reviewers and were independently screened for inclusion. If consensus was not reached through discussion between them, disagreements were solved by the decision of a third reviewer. Studies were considered eligible if they were able to answer the questions that have been defined according to the following PICO model:

1. (P) Participants: PwMS

2. (I) Intervention: Rehabilitation training with robotic-assisted device for upper limbs, with or without conventional therapy. 
3. (C) Comparator: Conventional rehabilitation.

4. (O) Outcome measures: Safety of robotic rehabilitation, the feasibility of robotic rehabilitation, upper limb strength, functioning, independence in activity of daily living (ADL), and Health-related Quality of Life (HRQoL).

We included systematic reviews, randomized controlled trials (RCTs), observational analytic studies, and crossover and case series. The exclusion criteria were (1) papers involving animals; (2) papers that had been written in language other than English; (3) a case report design; (4) participants with neurologic disabilities other than MS; and (5) robotic-assisted rehabilitation combined with other advanced technologies such as noninvasive brain stimulation (NIBS) compared to robot-assisted therapy alone.

\subsection{Data Extraction and Synthesis}

All of the data were extracted from eligible full-text documents through Excel by two different authors. In case of disagreement, a consensus was achieved by the review of a third author. The following data were extracted: (1) title; (2) authors; (3) publication year; (4) study design; (5) participants; (6) intervention characteristics; (7) outcomes; and (8) main findings. All of the studies that were included in the review were synthesized, describing both the study characteristics and the data that were extracted. Given the high clinical heterogeneity in design, intervention, and outcomes that were assessed in the different studies, a meta-analysis was not performed.

\subsection{Quality Assessment}

The study's risk of bias was assessed by the AMSTAR-2 [26] for systematic reviews and the 10-item PEDro scale [27] for RCT studies. Two different authors rated the studies that were included in this review. If consensus was not achieved after discussion, a third reviewer was interrogated. The 5-item Oxford CEBM scale was used to rate the level of evidence [28] (OCEBM website).

\section{Results}

After the databases were searched, 320 records were identified, of which 29 were assessed for full-text screening eligibility. Finally, ten studies were included in the qualitative analysis that verified the effect of robotic therapy in the upper limb rehabilitation in PwMS [16,29-37]. A summary of the search and study selection process is shown in Figure 1.

The ten selected studies included one systematic review [16]; four RCTs [29,30,32,33]; one randomized crossover study [35]; and four case series [31,34,36,37]. A summary of the characteristics and results of the included studies are reported in Table 1. 

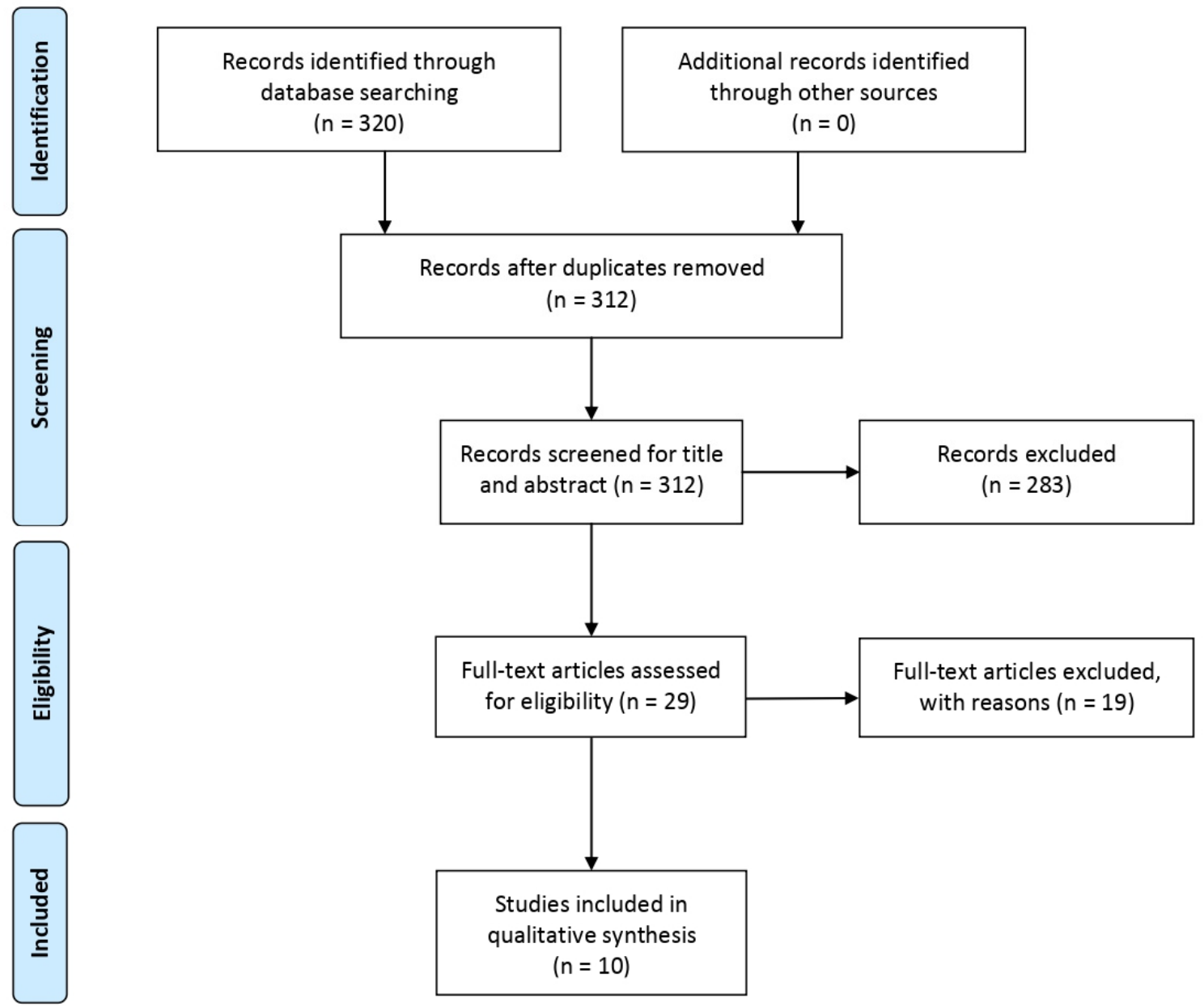

Figure 1. Flowchart of number of articles retrieved during the literature search and study selection.

Table 1. Main characteristics of the studies included in this review and related CEBM levels of evidence.

\begin{tabular}{|c|c|c|c|c|c|}
\hline Author, Year & Study Design & Population & Intervention & Outcomes & $\begin{array}{l}\text { CEBM Level } \\
\text { of Evidence }\end{array}$ \\
\hline $\begin{array}{c}\text { Solaro et al., } \\
2020\end{array}$ & RCT & $\begin{array}{l}36 \text { PwMS: } 13 \text { PP, } \\
15 \text { SP, 8 RR } \\
\text { EDSS 5-6 }\end{array}$ & $\begin{array}{c}\text { R: shoulder, elbow, forearm, wrist, } \\
\text { robot-assisted with haptic exercises } \\
\text { (Braccio di Ferro) } \\
\text { C: shoulder, elbow, forearm, wrist, } \\
\text { robot-assisted with sensorimotor exercises } \\
\text { (Braccio di Ferro) } \\
\text { D: } 8 \text { sessions, } 2 \text { sessions a week for } 4 \\
\text { weeks. Sessions lasted40 min. }\end{array}$ & $\begin{array}{l}(+) \text { 9HPT and robotic } \\
\text { instrumental outcomes } \\
\text { only for haptic group } \\
\text { no between-groups } \\
\text { differences }\end{array}$ & 2 \\
\hline $\begin{array}{l}\text { Gandolfi et al., } \\
2018\end{array}$ & RCT & $\begin{array}{l}44 \text { PwMS: } 3 \text { PP, } \\
15 \text { SP, } 26 \text { RR } \\
\text { EDSS } 6\end{array}$ & $\begin{array}{c}\text { R: hand robot-assisted training (Amadeo }{ }^{\circledR}, \\
\text { Tyromotion, Austria) } \\
\text { C: conventional therapy } \\
\text { D: } 10 \text { sessions, } 2 \text { sessions a week for } 5 \\
\text { weeks. Session lasting } 50 \text { min. }\end{array}$ & $\begin{array}{l}\text { (+) MAL, LifeH and } \\
\text { sEMG only for the } \\
\text { hand-robot group } \\
\text { no between-group } \\
\text { differences }\end{array}$ & 2 \\
\hline $\begin{array}{l}\text { Maris et al., } \\
\quad 2018\end{array}$ & Case Series & $\begin{array}{c}13 \text { PwMS: } 2 \text { PP, } 6 \\
\text { SP, } 3 \text { RR, } 2 \text { RP } \\
\text { EDSS } 6.5\end{array}$ & $\begin{array}{c}\text { R: shoulder, elbow, forearm, wrist } \\
\text { robot-assisted training (Haptic Master, } \\
\text { MOOG, Netherlands) } \\
\text { C: NA } \\
\text { D: } 40 \text { sessions, } 5 \text { times a week for } 8 \text { weeks. } \\
\text { Session lasting } 30 \text { min. }\end{array}$ & $\begin{array}{l}\text { (+) shoulder ROM, } \\
\text { handgrip strength, } \\
\text { WMFT, robotic } \\
\text { instrumental assessment }\end{array}$ & 4 \\
\hline
\end{tabular}


Table 1. Cont.

\begin{tabular}{|c|c|c|c|c|c|}
\hline Author, Year & Study Design & Population & Intervention & Outcomes & $\begin{array}{l}\text { CEBM Level } \\
\text { of Evidence }\end{array}$ \\
\hline $\begin{array}{l}\text { Sampson et al., } \\
2016\end{array}$ & Case Series & $\begin{array}{c}5 \text { PwMS: } 1 \text { PP, } 3 \\
\text { SP, } 1 \text { RR } \\
\text { EDSS NA }\end{array}$ & $\begin{array}{l}\text { R: shoulder, elbow, forearm, wrist training } \\
\text { (Armeo Spring, Hocoma, Switzerland) + } \\
\text { FES } \\
\text { C: NA } \\
\text { D: } 18 \text { treatment sessions over a } 10 \text { weeks } \\
\text { period. frequency not described }\end{array}$ & $\begin{array}{l}\text { (+) FMA proximal arm } \\
\text { section, the accuracy of } \\
\text { tracking performance }\end{array}$ & 4 \\
\hline Feys et al., 2015 & $\mathrm{RCT}$ & $\begin{array}{l}17 \text { PwMS: } 2 \text { PP, } \\
14 \text { SP, } 1 \text { RR } \\
\text { EDSS } 8\end{array}$ & $\begin{array}{c}\text { R: shoulder, elbow, forearm, wrist } \\
\text { robot-assisted training + conventional } \\
\text { multidisciplinary therapy (Haptic Master, } \\
\text { MOOG, Netherlands) } \\
\text { C: conventional multidisciplinary therapy } \\
\text { D: } 24 \text { sessions, } 3 \text { times a week for } 8 \text { weeks. } \\
\text { Session lasting } 30 \text { min of robotic therapy } \\
\text { and } 2 \text { h of conventional therapy. }\end{array}$ & $\begin{array}{l}\text { (+) patients' reported } \\
\text { beneficial changes in } \\
\text { daily use, robotic } \\
\text { instrumental assessment } \\
\text { No between-group } \\
\text { differences }\end{array}$ & 2 \\
\hline $\begin{array}{l}\text { Carpinella } \\
\text { et al., } 2012\end{array}$ & $\mathrm{RCT}$ & $\begin{array}{l}22 \text { PwMS: } 4 \text { PP, } \\
12 \text { SP, } 6 \text { RR } \\
\text { EDSS } 6.7\end{array}$ & $\begin{array}{c}\text { R: shoulder, elbow, forearm, wrist, } \\
\text { robot-assisted reaching task (Braccio di } \\
\text { Ferro) and object manipulation (RMT) } \\
\text { C: shoulder, elbow, forearm, wrist, } \\
\text { robot-assisted reaching task, RT (Braccio } \\
\text { di Ferro) } \\
\text { D: } 8 \text { sessions, } 160 \text { movements each session. } \\
\text { Session lasting 30-45 min. }\end{array}$ & $\begin{array}{l}(+) \text { grasp-ARAT, TSS } \\
\text { RMT was superior to RT } \\
\text { on grasp (ARAT) changes } \\
\qquad(p=0.035)\end{array}$ & 4 \\
\hline
\end{tabular}

R: shoulder, elbow, forearm, wrist passive training (Armeo Spring Hocoma

Gijbels et al., 2011

Case Series

9 PwMS: 3 PP, 6

SP

EDSS 7-8.5
(+) 9HPT, TEMPA

(+) TEMPA, ARAT at 2

months follow-up Switzerland) + usual care C: NA

D: 24 sessions, 3 times per week for 8 weeks, $30 \mathrm{~min} /$ session
4

R: shoulder, elbow, forearm, wrist robot-assisted training (Braccio di Ferro)

with error-enhancing (EE) forces

C: shoulder, elbow, forearm, wrist robot-assisted training (Braccio di Ferro) with error-reducing (ER)

D: 8 sessions (4 EE, 4 ER), 2 times a week for 4 weeks, 498 movements each session. Session lasting $60 \mathrm{~min}$.
(+) TADL in the first 4 sessions only in the EE group

No between-group differences
Cerebellar

deficits
R: shoulder, elbow, wrist shoulder, elbow, forearm, wrist, robot-assisted reaching task (Braccio di Ferro) C: NA

D: 8 sessions, 5 times a week, 200

movements each session.

$\begin{array}{lcc}\text { Carpinella } & \text { 7 PwMS: } 1 \text { PP, } 4 \\ \text { et al., 2009 } & \text { Case Series } & \text { SP, } 2 \text { RR } \\ & \text { EDSS } 4.5-6.5\end{array}$

(+) 9HPT and robotic instrumental assessment

\begin{tabular}{|c|c|c|c|c|c|}
\hline $\begin{array}{l}\text { Lamers et al., } \\
\qquad 2016\end{array}$ & $\begin{array}{l}\text { Systematic } \\
\text { Review }\end{array}$ & $\begin{array}{l}41 \text { PwMS (4 } \\
\text { studies) }\end{array}$ & $\begin{array}{c}30 \text { included studies, of which only } 4 \text { used } \\
\text { robotics }\end{array}$ & $\begin{array}{l}\text { Robotic training is the } \\
\text { most studied } \\
\text { rehabilitation strategy in } \\
\text { the field of upper limb } \\
\text { rehabilitation in PwMS. } \\
\text { The included studies } \\
\text { (RCT and non-controlled } \\
\text { trials) have shown that } \\
\text { robotic training may } \\
\text { improve motor } \\
\text { coordination, manual } \\
\text { dexterity and upper limb } \\
\text { functionality in PwMS. }\end{array}$ & 2 \\
\hline
\end{tabular}

${ }^{1}$ PwMS = patients with multiple sclerosis; $\mathrm{PP}=$ primary progressive; $\mathrm{SP}=$ secondary progressive; $\mathrm{RR}=$ relapsingremitting; $\mathrm{RP}=$ relapsing-progressive; $\mathrm{EDSS}=$ expanded disability status scale; $\mathrm{R}=$ robotics group; $\mathrm{C}=\mathrm{control}$ group; $\mathrm{D}=$ dose; $9 \mathrm{HPT}=$ nine hole peg test; ARAT = action research arm test; FMA = fugl-meyer assessment; $\mathrm{MAL}=$ motor activity $\log ; \mathrm{TADL}=$ tremor in activity of daily life; TSS = tremor severity scale; LifeH = life habits assessment; sEMG = surface electromyography; WMFT = wolf motor function test; RT = reaching tasks; RMT $=$ reaching and manipulation tasks; $\mathrm{EE}=$ error-enhancing; $\mathrm{RCT}=$ randomized controlled trial; $(+)=$ robotic within-group significant difference. 
In the studies that reported this information [30,32,37], the most affected arm was usually selected for robot-assisted training. In PwMS, both end-effectors [29,32,33,35-37], a passive exoskeleton [31,34], and a hand robot [30] have been used in rehabilitation so far. The Braccio di Ferro, a planar robotic manipulandum with 2 degrees of freedom that can assist or contrast patients during a reaching task with either resistive, assistive, or perturbating force fields was the most tested device in this population $[29,33,35,36]$. The Amadeo $^{\circledR}$ (Tyromotion-Austria), a hand robotic device with each finger attached to the robotically driven slide, was used in a recent RCT [30]. The HapticMaster robot (MOOG, the Netherlands), an end-effector with a 3D workspace for upper limb training, was used in a case series and an RCT study [32,37]. Finally, the Armeo Spring (Hocoma AG, Zurich, $\mathrm{CH}$ ), a passive rehabilitation exoskeleton with sensors for the shoulder, elbow, and wrist joints with gravity support for the arm in a virtual learning environment, was used in two case series studies [31,34].

Overall, 161 PwMS (EDSS 4.5-8; 29 primary progressive, 81 secondary progressive, 49 relapsing-remitting, and 2 relapsing-progressive) were included in the clinical studies. The RCTs included, respectively, 22 [33], 17 [32], 44 [30], and 36 [29] patients. Eight patients were included in the crossover study [35], and the remaining 34 patients were included in the four case series studies [31,34,36,37]. The systematic review [16] that only considered robotic studies included 41 patients. Considering the included studies, the duration of the robotic treatment varied from 30 to $60 \mathrm{~min}$. The frequency of the therapy was about 2-5 per week for 4-8 weeks, depending on the study, with a total number of sessions that ranged between 8 and 40. Only one study [32] associated the robotic treatment to the conventional one, whereas conventional therapy [30], the adjunction of object manipulation to robotassisted reaching tasks [33] or sensorimotor training [29] were selected as comparators. Within-group significant differences in the robotic group were highlighted for the following outcome measures: handgrip strength; active range of movement (ROM); Nine Hole Peg Test (9HPT) for dexterity; Wolf Motor Function Test (WMFT) and Action Research Arm Test (ARAT) for arm function; Tremor in Activity of Daily Life (TADL) questionnaire and Tremor Severity Scale (TSS) for tremor; Fugl-Meyer assessment Scale (FMA) for upper limb impairment; Life Habits assessment—general short form (LifeH), Motor Activity Log (MAL) for upper limb use; and TEMPA for arm function during daily living activities. In addition to clinical outcome measures, a set of robotic-driven measurements for motor control and surface electromyography upper limb muscle activity were included in the analysis.

\subsection{Effects of Upper Limb Robot-Assisted Therapy}

The included systematic review [16] highlighted that robotic training is the most studied rehabilitation intervention in upper limb rehabilitation in PwMS. In general, the included studies (RCTs and non-RCTs) have shown that robotic training can improve the motor coordination, manual dexterity, and functional capacity of the upper limbs. At the body function level, maximum shoulder anteflexion (+27 degrees) and handgrip strength $(+12 \%)$ were increased in a prospective cohort study [37]. In contrast, a case series study reported an improvement in proximal arm impairment $(+15 \%)$ [31]. The tremor was decreased after an error-enhancing adaptive robotic protocol [35]. Several studies reported improving robot-driven measures [29,32,36,37] or EMG activity [30], but this was only the case in the robotic group. At the activity level, manual dexterity, which was measured by $9 \mathrm{HPT}$, improved by $12-47 \mathrm{~s}$ in two case series studies $[34,36]$ and only in the robotic group (+15\%) in an RCT [29]. Arm function, which was measured by WMFT, increased by $25-33 \%$ in a prospective cohort study [37]; however, the ARAT Grasp subscore significantly improved in the robotic protocol that included real objects manipulation $(+77 \%)$ compared to the other condition (+29\%) [33]. When measured by TEMPA tasks, arm capacity improved (-23 s) in a case series study [34]; however, better arm use in everyday life was only reported in the robotic group in two RCTs [30,32]. Only four studies $[29,30,34,37]$ had a follow-up evaluation, with retention of arm function being shown to be maintained at one [30] or two months [34] after robotic therapy. 


\subsection{Quality Assessment}

The PEDro scale assessment reported that the RCTs had high quality overall $[29,30$, 32,33,35]. In contrast, the AMSTAR-2 assessment highlighted that the review that was included [16] had a critically low quality. The complete quality assessment is reported in Table 2 (PEDro) and in Table 3 (AMSTAR-2).

Table 2. Quality assessment of the RCT studies that were included using the PEDro Checklist.

\begin{tabular}{|c|c|c|c|c|c|c|c|c|c|c|c|c|}
\hline Author, Year & Q1 & Q2 & Q3 & Q4 & Q5 & Q6 & Q7 & Q8 & Q9 & Q10 & Q11 & PEDro Score \\
\hline C. Solaro et al., 2020 & $\mathrm{Y}$ & Y & $\mathrm{N}$ & $\mathrm{N}$ & $\mathrm{Y}$ & $\mathrm{N}$ & $\mathrm{Y}$ & $\mathrm{Y}$ & $\mathrm{N}$ & $\mathrm{Y}$ & $\mathrm{Y}$ & 6 \\
\hline Gandolfi et al., 2018 & $\mathrm{Y}$ & $\mathrm{Y}$ & $\mathrm{Y}$ & $\mathrm{Y}$ & $\mathrm{N}$ & $\mathrm{N}$ & $\mathrm{Y}$ & Y & $\mathrm{N}$ & $\mathrm{Y}$ & $\mathrm{Y}$ & 7 \\
\hline Feys et al., 2015 & $\mathrm{Y}$ & $\mathrm{Y}$ & $\mathrm{Y}$ & $\mathrm{Y}$ & $\mathrm{N}$ & $\mathrm{N}$ & $\mathrm{N}$ & $\mathrm{Y}$ & $\mathrm{Y}$ & $\mathrm{Y}$ & $\mathrm{Y}$ & 7 \\
\hline Carpinella et al., 2012 & $\mathrm{Y}$ & $\mathrm{Y}$ & $\mathrm{N}$ & $\mathrm{N}$ & $\mathrm{N}$ & $\mathrm{Y}$ & $\mathrm{Y}$ & $\mathrm{Y}$ & $\mathrm{Y}$ & $\mathrm{Y}$ & $\mathrm{Y}$ & 7 \\
\hline Vergaro et al., 2010 & $\mathrm{Y}$ & $\mathrm{Y}$ & $\mathrm{N}$ & $\mathrm{N}$ & $\mathrm{Y}$ & $\mathrm{N}$ & $\mathrm{Y}$ & $\mathrm{Y}$ & $\mathrm{Y}$ & $\mathrm{Y}$ & $\mathrm{Y}$ & 7 \\
\hline
\end{tabular}

Table 3. Quality assessment of the systematic review that was included using the AMSTAR 2 criteria.

\begin{tabular}{|c|c|c|c|c|c|c|c|c|c|c|c|c|c|c|c|c|c|}
\hline $\begin{array}{l}\text { Author, } \\
\text { Year }\end{array}$ & Q1 & Q2 & Q3 & Q4 & Q5 & Q6 & Q7 & Q8 & Q9 & Q10 & Q11 & Q12 & Q13 & Q14 & Q15 & Q16 & $\begin{array}{c}\text { AMSTAR } \\
2 \text { Score }\end{array}$ \\
\hline $\begin{array}{l}\text { Lamers } \\
\text { et al., } 2016\end{array}$ & Y & $\mathrm{N}$ & $\mathrm{N}$ & PY & Y & Y & $\mathrm{N}$ & PY & $\mathrm{N}$ & $\mathrm{N}$ & NMC & $\mathrm{NMC}$ & $\mathrm{N}$ & $\mathrm{N}$ & NMC & $\mathrm{Y}$ & $\begin{array}{c}\text { Critically } \\
\text { low } \\
\text { quality }\end{array}$ \\
\hline
\end{tabular}

Abbreviations: $\mathrm{Y}=$ Yes, $\mathrm{N}=\mathrm{No}, \mathrm{PY}=$ partial yes, $\mathrm{NMC}=$ no meta-analysis conducted.

\subsection{Levels of Evidence}

An evidence Level of 2 was assigned to the systematic review [16] and three RCTs [29,30,32]; a level of 4 was assigned to the other studies [31,33-37].

\subsection{Adverse Effects}

Five of the included studies $[30,31,33,34,37]$ considered RAT safety, reporting no adverse effects related to the intervention.

\section{Discussion}

Upper limb impairment in PwMS is often under-recognized, even if it is related to performing everyday activities that are crucial for a patient to maintain their quality of life and participation in daily activities [11,38]. For this reason, necessary is necessary to implement and develop effective rehabilitation strategies. This evidence-based review aimed to systematically report the state-of-the art on clinical applications and the effectiveness of robotic-assisted upper limb rehabilitation in PwMS. So far, four robotic devices have been tested in PwMS: two end-effectors (Braccio di Ferro, HapticMaster), a passive gravity-supported exoskeleton (Armeo Spring), and a hand-robot (Amadeo). Both the HapticMaster and Armeo Spring training movements were tested in a 3D virtual environment.

The end-effector, called Braccio di Ferro, was tested in two RCTs [29,33], one randomized crossover study [35], and one case series [36]. The adaptive-type robotic therapy for the upper limbs was feasible in PwMS and was helpful in improving manual dexterity $[35,36]$ even though no clear conclusion has been made on the best approach to improve motor coordination in patients with cerebellar deficits [35]. Furthermore, Carpinella et al. demonstrated in a later study that a robotic training protocol that includes reaching tasks and object manipulation may improve movements that involve the grasping function compared to a training protocol that only provides reaching tasks [33]. A recent paper [29] used this device to compare robot-assisted training based on a haptic or sensorimotor exercise in an 8-session protocol ( 2 per week). Patients in the haptic group had to counteract incoordination and weakness when interacting with a virtual mass-spring system against a 
resistive load. Instead, subjects in the sensorimotor group performed reaching movements under visual control. This study showed that haptic protocol proved to be slightly superior (without between-group differences) to sensorimotor treatment in improving upper limb dexterity. This could be related to amelioration in upper limb and shoulder coordination. These improvements are most evident in prevalent pyramidal impaired subjects who were unable to maintain the effects of rehabilitation after three months [29].

The robotic training with the Haptic Master Robot was used in an RCT [32] and in a prospective cohort study [37]. In addition to traditional therapy, it showed significant improvements in the quality of the movement execution in wheelchair-bound populations and demonstrated reported beneficial effects on daily use without any adjunct impact on clinical outcome measures [32]. By increasing the number of sessions (from 3 to 5 weeks) and by utilizing the intervention on a less impaired population [37], significant improvements were also noted in clinical parameters such as active ROM in the shoulder and the strength and motor capacity of the upper limbs, unlike in stroke patients [37].

Two case series studies explored the effects of Armeo Spring. An 8-week long training program (3 times per week for $30 \mathrm{~min} /$ session) that was implemented supplementary to conventional therapy in high-level disability PwMS (EDSS 7.0-8.5) was able to improve the functional capacity of the upper limbs, even after a 2-month follow-up [34]. Sampson combined the use of the Armeo Spring with functional electric stimulation (FES). This system assisted patients in following a specified trajectory path, employing an iterative learning control to improve accuracy and maximize voluntary effort. An improvement of the proximal upper limb was reported in a small group who underwent a training program for 18 treatment sessions over 10 weeks [31]. Finally, an RCT did not find the superiority of the hand-robot Amadeo on conventional therapy for clinical measures, even if only the robot-assisted group reported significant improvement in arm use and an enhancement in extensor carpi activation [30].

In conclusion, end-effector robotic devices may represent a therapeutic opportunity, improving manual dexterity, coordination, functional capacity, and the efficiency of upper limb motor strategies in PwMS [29,30,32,33,36,37]. However, the literature does not highlight statistically significant benefits of RAT compared to [30] or in addition to conventional rehabilitation [32]. Most of the included studies show that the clinical assessment does not always confirm improvements in instrumental measurements. A possible explanation could be related to the fact that kinematic and motor activity impairments can be present with a normal upper limb function [39]. Moreover, robotic-driven measurements can offer a more detailed picture of the execution of everyday daily living activities [40]. The included studies highlight that the improvements are not always maintained until the follow-up, except for arm function $[30,34]$, probably because there may be no automatic transfer of the improved task performance to the overall upper limb functionality. The use of real objects during purposeful, functional tasks involving the whole upper limb can be a good solution to generalize motor tasks to daily living activities. In this direction, using a planar robot with real object manipulation has effectively ameliorated grasping function in PwMS [33].

Robot-assisted therapy has been proposed to induce neuroplastic changes and motor recovery mainly through two different paradigms: adaptive or assistive [18]. Even though the assistive approach is usually the most commonly applied approach in arm robotic therapy, the evidence in PwMS is more focused on the adaptive approach with a solid neurophysiological rationale $[29,33,35,36]$. This is based on the idea that PwMS preserve their ability to adapt to a novel dynamic environment, an ability that is related to the feed-forward component of control $[9,36]$. The robot did not assist subjects during the movement execution but provided an unfamiliar dynamic environment. The subjects are required to adapt by learning to predict the effects of perturbating forces. However, even if motor adaptation occurred when robotic force fields were applied [35], a recent RCT did not drive definite conclusions on the superiority of this adaptive protocol compared to during sensorimotor training using the same device [29]. Conversely, a decrease in tremor and cerebellar deficits was only observed when an error-enhancing paradigm was 
proposed, leading to the hypothesis that this population would be responsive to errorbased learning [35].

\section{Limits and Future Directions}

No meta-analysis that considered the different outcomes used, types of robotic protocols, or included patients with different arm impairments concerning severity or predominant motor pattern (weakness, incoordination) was performed on the five RCTs that were included in this evidence-based review. Moreover, due to the heterogeneity of treatment protocols in terms of duration and frequency, it is currently not possible to demonstrate how many weekly sessions or which therapy duration could lead to the best rehabilitative results. In addition, all of the studies analyzed the use of end-effector robots and did not consider the use of robotic exoskeletons, which are still not widespread in clinical practice. The reported studies also did not report on the cost-benefit ration or the evidence of efficiency. More RCTs with larger sample sizes and follow-up evaluations are required. Finally, MS-specific characteristics should be addressed more deeply in future studies. As previously reported, arm impairment is usually bilateral and usually represents different motor deficits (weakness/incoordination, learned non-use). Future studies would be more targeted on a specific impairment, and it is likely that a bilateral robotic arm approach would be helpful in this population. Moreover, even if severely disabled patients are more likely to be candidates for arm rehabilitation, functional reorganization processes could be limited by accumulating structural CNS damage [41]. It would also be essential to implement specific protocols for PwMS in terms of duration, intensity, dose, and cognitive-motor dysfunction according to the stage and type of the disease.

\section{Conclusions}

Even if arm impairment is widespread and can dramatically reduce functional abilities and participation in PwMS, the current evidence on the use of robot-assisted therapy in this population is still lacking and is only based on few high-quality RCTs and other uncontrolled pilot studies. Robot-assisted treatment seems safe and useful to increase manual dexterity and the quality of movement execution in PwMS with moderate to severe disability. Additional studies with an adequate sample size and methodological rigour are warranted to drive definite conclusions.

Author Contributions: G.M., M.T., E.F.R., I.A. and S.S. (Sofia Straudi) conceptualized the paper, performed, and/or supervised the research. M.T., S.S. (Sofia Straudi) and E.F.R. drafted the manuscript. L.P., M.A., M.G. (Marialuisa Gandolfi), M.P. (Matteo Paci), E.C. (Emanuela Casanova), D.M., G.L.R., F.B., S.S. (Silvia Sterzi), D.G., A.B., S.M. (Sandra Miccinilli), S.F., M.S. (Monica Sicari), S.P., C.M.S., S.G. (Stefano Gargano) P.B. (Paolo Benanti), P.B. (Paolo Boldrini), D.B., E.C. (Enrico Castelli), F.D., V.F., S.G. (Silvia Galeri), F.G., M.G. (Mauro Grigioni), S.M. (Stefano Mazzoleni), S.M. (Stefano Mazzon), F.M., M.P. (Maurizio Petrarca), A.P., F.P., M.S. (Michele Senatore) and G.T., members of the organizing committee and the scientific and technical committees of the CICERONE consensus conference, supervised the research, read and corrected the manuscript. All authors have read and agreed to the published version of the manuscript.

Funding: The authors report no involvement in the research by the sponsor that could have influenced the outcome of this work.

Institutional Review Board Statement: Not applicable.

Informed Consent Statement: Not applicable.

Data Availability Statement: The dataset is available on request.

Acknowledgments: The present study has been carried out within the framework of the Italian Consensus Conference on "Rehabilitation assisted by robot and electromechanical devices for persons with disability of neurological origin" (CICERONE), promoted by the Italian Society of Physical and Rehabilitation Medicine (SIMFER, Società Italiana di Medicina Fisica e Riabilitativa) and Italian 
Society of Neurological Rehabilitation (SIRN, Società Italiana di Riabilitazione Neurologica) (2019_ 2021).

Conflicts of Interest: The authors declare no conflict of interest.

Group Name: Upper limb working group within the "Italian Consensus Conference on rehabilitation assisted by robotic and electromechanical devices for persons with disability of neurological origin" (CICERONE): Michela AGOSTINI (Department of Neuroscience, section of rehabilitation, UniversityGeneral Hospital of Padova, Italy); Irene APRILE (IRCCS Fondazione Don Carlo Gnocchi ONLUS Florence, Italy); Chiara Arienti (IRCCS Fondazione Don Carlo Gnocchi ONlus, Milan, Italy), Ludovica BALUARDO (Ferrara University Hospital, Neuroscience and Rehabilitation Department, Ferrara, Italy); Alberto BATTISTINI (IRCCS Istituto delle Scienze Neurologiche di Bologna, UOC Medicina Riabilitativa e Neuroriabilitazione, Bologna, Italia); Paolo BENANTI (Pontifical Gregorian University, Rome, Italy); Valentina BOETTO (Università degli studi di Torino); Paolo BOLDRINI (Società Italiana di Medicina Fisica e Riabilitativa, SIMFER); Donatella BONAIUTI (Istituto Società Italiana di Medicina Fisica e Riabilitativa, SIMFER); Marco BRAVI (Biomedical Campus University Foundation, Rome, Italy); Federica BRESSI (Biomedical Campus University Foundation, Rome, Italy); Diletta BRUNO (Biomedical Campus University Foundation, Rome, Italy); Emanuela CASANOVA (IRCCS Istituto delle Scienze Neurologiche di Bologna, UOC Medicina Riabilitativa e Neuroriabilitazione, Bologna, Italia); Enrico CASTELLI (Paediatric Neurorehabilitation, Bambino Gesù Children's Hospital, Rome, Italy); Manuela DESILVESTRI (Neurorehabilitation Unit, Azienda Ospedaliera SS. Antonio e Biagio e Cesare Arrigo, Alessandria, Italy, EU); Francesco DRAICCHIO (Department of Occupational and Environmental Medicine, Epidemiology and Hygiene, INAIL, Monte Porzio Catone, 00185 Rome, Italy); Vincenzo FALABELLA (President Italian Federation of Persons with Spinal Cord Injuries (Faip Onlus), Rome, Italy); Serena FILONI (Padre Pio Foundation and Rehabilitation Centers, San Giovanni Rotondo, Italy); Silvia GALERI (IRCCS Fondazione Don Carlo Gnocchi, Milan, Italy); Marialuisa GANDOLFI (Department of Neurosciences, Biomedicine and Movement Sciences, University of Verona, Verona, Italy); Stefano GARGANO (Fondazione Don Carlo Gnocchi-Torino); Daniele GIANSANTI (National Center for Innovative Technologies in Public Health, Italian National Institute of Health, Rome, Italy); Francesca GIMIGLIANO (Department of Mental and Physical Health and Preventive Medicine, University of Campania “Luigi Vanvitelli”, Naples, Italy); Mauro GRIGIONI (National Center for Innovative Technologies in Public Health, Italian National Institute of Health, Rome, Italy); Giuseppe LA ROSA (C.S.R.-Consorzio Siciliano di Riabilitazione, Catania, Italy); Dario MARINO (IRCCS Neurolysis Center "Bonino Pulejo", Messina, Italy); Alex MARTINO CINNERA (Santa Lucia Foundation, IRCCS, Rome, Italy); Stefano MAZZOLENI (Department of Electrical and Information Engineering, Politecnico di Bari, Italy); Stefano MAZZON (AULSS6 (Unique Sanitary Local Company) Euganea Padova-Rehabilitation Department); Sandra MICCINILLI (Biomedical Campus University Foundation, Rome, Italy); Franco MOLTENI (Villa Beretta, Costa Masnaga, Italy); Giovanni MORONE (Santa Lucia Foundation, IRCCS, Rome, Italy); Matteo PACI (AUSL (Unique Sanitary Local Company, District of Central Tuscany, Florence, Italy); Angela PALOMBA (Department of Medical and Surgical Specialties and Dentistry, University of Campania "Luigi Vanvitelli", Naples, Italy 31); Cristiano PECCHIOLI (Department of Neuroscience and Neurorehabilitation, IRCCS San Raffaele Pisana, Rome, Italy); Luca PERRERO (Neurorehabilitation Unit, Azienda Ospedaliera SS. Antonio e Biagio e Cesare Arrigo, Alessandria, Italy); Maurizio PETRARCA ("Bambino Gesù" Children's Hospital-IRCCS, Movement Analysis and Robotics Laboratory MARlab, Rome, Italy); Salvatore PETROZZINO (A.O.U. Città della Salute e della Scienza di Torino, Torino, Italy); Alessandro PICELLI (Department of Neurosciences, Biomedicine and Movement Sciences, University of Verona, Verona, Italy); Federico POSTERARO (Versilia Hospital AUSL Toscana Nord Ovest, Italy); Emanuele Francesco RUSSO (Padre Pio Foundation and Rehabilitation Centers, San Giovanni Rotondo, Italy); Fabio SANTACATERINA (Biomedical Campus University Foundation, Rome, Italy); Michele SENATORE (Past President A.I.T.O. (Associazione Italiana Terapisti Occupazionali); Monica SICARI (A.O.U. Città della Salute e della Scienza di Torino, Torino, Italy); Claudio Marcello SOLARO (CRRF "Mons. Luigi Novarese" Moncrivello, VC, Italy); Silvia STERZI (Biomedical Campus University Foundation, Rome, Italy); Sofia STRAUDI (Ferrara University Hospital, Neuroscience and Rehabilitation Department, Ferrara, Italy); Giuseppe TURCHETTI (Management Institute, Sant'Anna School of Advanced Studies, Pisa, Italy). 


\section{References}

1. Compston, A.; Coles, A. Multiple sclerosis. Lancet 2008, 372, 1502-1517. [CrossRef] [PubMed]

2. Compston, A.; Coles, A. Multiple sclerosis. Lancet 2002, 359, 1221-1231. [CrossRef]

3. Johansson, S.; Ytterberg, C.; Claesson, I.M.; Lindberg, J.; Hillert, J.; Andersson, M.; Holmqvist, L.W.; von Koch, L. High concurrent presence of disability in multiple sclerosis Associations with perceived health. J. Neurol. 2007, 254, 767-773. [CrossRef]

4. Thompson, A.J. Progress in neurorehabilitation in multiple sclerosis. Curr. Opin. Neurol. 2002, 15, 267-270. [CrossRef]

5. Koch, M.; Mostert, J.; Heersema, D.; De Keyser, J. Tremor in multiple sclerosis. J. Neurol. 2007, 254, 133-145. [CrossRef]

6. Bertoni, R.; Lamers, I.; Chen, C.C.; Feys, P.; Cattaneo, D. Unilateral and bilateral upper limb dysfunction at body functions, activity and participation levels in people with multiple sclerosis. Mult. Scler. J. 2015, 21, 1566-1574. [CrossRef] [PubMed]

7. Lamers, I.; Cattaneo, D.; Chen, C.C.; Bertoni, R.; Van Wijmeersch, B.; Feys, P. Associations of upper limb disability measures on different levels of the international classification of functioning, disability and health in people with multiple sclerosis. Phys. Ther. 2015, 95, 65-75. [CrossRef]

8. Pellegrino, L.; Coscia, M.; Muller, M.; Solaro, C.; Casadio, M. Evaluating upper limb impairments in multiple sclerosis by exposure to different mechanical environments. Sci. Rep. 2018, 8, 2110. [CrossRef]

9. Casadio, M.; Sanguineti, V.; Morasso, P.; Solaro, C. Abnormal sensorimotor control, but intact force field adaptation, in multiple sclerosis subjects with no clinical disability. Mult. Scler. J. 2008, 14, 330-342. [CrossRef] [PubMed]

10. Miller, D.M.; Allen, R. Quality of life in multiple sclerosis: Determinants, measurement, and use in clinical practice. Curr. Neurol. Neurosci. Rep. 2010, 10, 397-406. [CrossRef]

11. Cattaneo, D.; Lamers, I.; Bertoni, R.; Feys, P.; Jonsdottir, J. Participation restriction in people with multiple sclerosis: Prevalence and correlations with cognitive, walking, balance, and upper limb impairments. Arch. Phys. Med. Rehabil. 2017, 98, 1308-1315. [CrossRef]

12. Kierkegaard, M.; Einarsson, U.; Gottberg, K.; Von Koch, L.; Holmqvist, L.W. The relationship between walking, manual dexterity, cognition and activity/participation in persons with multiple sclerosis. Mult. Scler. J. 2011, 18, 639-646. [CrossRef] [PubMed]

13. Tomassini, V.; Matthews, P.M.; Thompson, A.; Fuglø, D.; Geurts, J.J.; Johansen-Berg, H.; Jones, D.K.; Rocca, M.A.; Wise, R.G.; Barkhof, F.; et al. Neuroplasticity and functional recovery in multiple sclerosis. Nat. Rev. Neurol. 2012, 8, 635-646. [CrossRef]

14. Tomassini, V.; Johansen-Berg, H.; Leonardi, L.; Paixão, L.; Jbabdi, S.; Palace, J.; Pozzilli, C.; Matthews, P.M. Preservation of motor skill learning in patients with multiple sclerosis. Mult. Scler. J. 2010, 17, 103-115. [CrossRef] [PubMed]

15. Feys, P.; Straudi, S. Beyond therapists: Technology-aided physical MS rehabilitation delivery. Mult. Scler. J. 2019, 25, 1387-1393. [CrossRef] [PubMed]

16. Morone, G.; Cocchi, I.; Paolucci, S.; Iosa, M. Robot-assisted therapy for arm recovery for stroke patients: State of the art and clinical implication. Expert Rev. Med. Devices 2020, 17, 223-233. [CrossRef]

17. Turner, D.L.; Murguialday, A.R.; Birbaumer, N.; Hoffmann, U.; Luft, A. Neurophysiology of robot-mediated training and therapy: A perspective for future use in clinical populations. Front. Neurol. 2013, 4, 184. [CrossRef]

18. Huang, V.S.; Krakauer, W. Robotic neurorehabilitation. A computational motor learning prospective. J. Neuroeng. Rehabil. 2009, 6 , 5. [CrossRef]

19. Mehrholz, J.; Pohl, M.; Platz, T.; Kugler, J.; Elsner, B. Electromechanical and robot-assisted arm training for improving activities of daily living, arm function, and arm muscle strength after stroke. Cochrane Database Syst. Rev. 2018, 9, CD006876. [CrossRef]

20. Morone, G.; Palomba, A.; Martino Cinnera, A.; Agostini, M.; Aprile, I.; Arienti, C.; Paci, M.; Casanova, E.; Marino, D.; LARosa, G.; et al. Systematic review of guidelines to identify recommendations for upper limb robotic rehabilitation after stroke. Eur. J. Phys. Rehabil. Med. 2021, 57, 238-245. [CrossRef]

21. Yeh, S.-W.; Lin, L.-F.; Tam, K.-W.; Tsai, C.-P.; Hong, C.-H.; Kuan, Y.-C. Efficacy of robot-assisted gait training in multiple sclerosis: A systematic review and meta-analysis. Mult. Scler. Relat. Disord. 2020, 41, 102034. [CrossRef] [PubMed]

22. Spooren, A.I.; Timmermans, A.A.; Seelen, H.A. Motor training programs of arm and hand in patients with MS according to different levels of the ICF: A systematic review. BMC Neurol. 2012, 2, 12-49. [CrossRef] [PubMed]

23. Lamers, I.; Maris, A.; Severijns, D.; Dielkens, W.; Geurts, S.; Van Wijmeersch, B.; Feys, P. Upper limb rehabilitation in people with multiple sclerosis. Neurorehabilit. Neural Repair 2016, 30, 773-793. [CrossRef] [PubMed]

24. Gimigliano, F.; Palomba, A.; Arienti, C.; Morone, G.; Perrero, L.; Agostini, M.; Aprile, I.; Paci, M.; Casanova, E.; Marino, D.; et al. Robot-assisted arm therapy in neurological health conditions: Rationale and methodology for the evidence synthesis in the CICERONE Italian Consensus Conference. Eur. J. Phys. Rehabil. Med. 2021, 57, 824-830. [CrossRef]

25. Moher, D.; Liberati, A.; Tetzlaff, J.; Altman, D.G.; PRISMA Group. Preferred reporting items for systematic reviews and meta-analyses: The PRISMA statement. BMJ 2009, 339, b2535. [CrossRef] [PubMed]

26. Shea, B.J.; Reeves, B.C.; Wells, G.; Thuku, M.; Hamel, C.; Moran, J.; Moher, D.; Tugwell, P.; Welch, V.; Kristjansson, E.; et al. AMSTAR 2: A critical appraisal tool for systematic reviews that include randomised or non-randomised studies of healthcare interventions, or both. BMJ 2017, 358, j4008. [CrossRef]

27. Moseley, A.M.; Herbert, R.; Sherrington, C.; Maher, C.G. Evidence for physiotherapy practice: A survey of the Physiotherapy Evidence Database (PEDro). Aust. J. Physiother. 2002, 48, 43-49. [CrossRef]

28. Durieux, N.; Vandenput, S.; Pasleau, F. OCEBM levels of evidence system. Rev. Med. Liege 2013, 68, 644-649. [PubMed] 
29. Solaro, C.; Cattaneo, D.; Basteris, A.; Carpinella, I.; De Luca, A.; Mueller, M.; Bertoni, R.; Ferrarin, M.; Sanguineti, V. Haptic vs sensorimotor training in the treatment of upper limb dysfunction in multiple sclerosis: A multi-center, randomized controlled trial. J. Neurol. Sci. 2020, 412, 116743. [CrossRef]

30. Gandolfi, M.; Valè, N.; Dimitrova, E.K.; Mazzoleni, S.; Battini, E.; Benedetti, M.D.; Gajofatto, A.; Ferraro, F.; Castelli, M.; Camin M.; et al. Effects of high-intensity robot-assisted hand training on upper limb recovery and muscle activity in individuals with multiple sclerosis: A randomized, controlled, single-blinded trial. Front. Neurol. 2018, 9, 905. [CrossRef] [PubMed]

31. Sampson, P.; Freeman, C.; Coote, S.; Demain, S.; Feys, P.; Meadmore, K.; Hughes, A.-M. Using functional electrical stimulation mediated by iterative learning control and robotics to improve arm movement for people with multiple sclerosis. IEEE Trans. Neural Syst. Rehabil. Eng. 2016, 24, 235-248. [CrossRef] [PubMed]

32. Feys, P.; Coninx, K.; Kerkhofs, L.; De Weyer, T.; Truyens, V.; Maris, A.; Lamers, I. Robot-supported upper limb training in a virtual learning environment: A pilot randomized controlled trial in persons with MS. J. Neuroeng. Rehabil. 2015, 12, 60. [CrossRef] [PubMed]

33. Carpinella, I.; Cattaneo, D.; Bertoni, R.; Ferrarin, M. Robot training of upper limb in mu tiple sclerosis: Comparing protocols with or without manipulative task components. IEEE Trans. Neural Syst. Rehabil. Eng. 2012, 20, 351-360. [CrossRef]

34. Gijbels, D.; Lamers, I.; Kerkhofs, L.; Alders, G.; Knippenberg, E.; Feys, P. The Armeo Spring as training tool to improve upper limb functionality in multiple sclerosis: A pilot study. J. Neuroeng. Rehabil. 2011, 8, 5. [CrossRef] [PubMed]

35. Vergaro, E.; Squeri, V.; Brichetto, G.; Casadio, M.; Morasso, P.; Solaro, C.; Sanguineti, V. Adaptive robot training for the treatment of incoordination in Multiple Sclerosis. J. Neuroeng. Rehabil. 2010, 7, 37. [CrossRef] [PubMed]

36. Carpinella, I.; Cattaneo, D.; Abuarqub, S.; Ferrarin, M. Robot-based rehabilitation of the upper limbs in multiple sclerosis: Feasibility and preliminary results. J. Rehabil. Med. 2009, 41, 966-970. [CrossRef] [PubMed]

37. Maris, A.; Coninx, K.; Seelen, H.; Truyens, V.; De Weyer, T.; Geers, R.; Lemmens, M.; Coolen, J.; Stupar, S.; Lamers, I.; et al. The impact of robot-mediated adaptive I-TRAVLE training on impaired upper limb function in chronic stroke and multiple sclerosis. Disabil. Rehabil. Assist. Technol. 2018, 13, 1-9. [CrossRef]

38. Marrie, R.A.; Cutter, G.R.; Tyry, T.; Cofield, S.S.; Fox, R.; Salter, A. Upper limb impairment is associated with use of assistive devices and unemployment in multiple sclerosis. Mult. Scler. Relat. Disord. 2017, 13, 87-92. [CrossRef]

39. Solaro, C.; Brichetto, G.; Casadio, M.; Roccatagliata, L.; Ruggiu, P.; Mancardi, G.L.; Morasso, P.; Tanganelli, P.; Sanguineti, V. Subtle upper limb impairment in asymptomatic multiple sclerosis subjects. Mult. Scler. J. 2007, 13, 428-432. [CrossRef]

40. Pellegrino, L.; Stranieri, G.; Tiragallo, E.; Tacchino, A.; Brichetto, G.; Coscia, M.; Casadio, M. Analysis of upper limb movement in Multiple Sclerosis subjects during common daily actions. Annu. Int. Conf. IEEE Eng. Med. Biol. Soc. 2015, 2015, 6967-6970. [CrossRef]

41. Schoonheim, M.M.; Geurts, J.J.; Barkhof, F. The limits of functional reorganization in multiple sclerosis. Neurology 2010, 74, 1246-1247. [CrossRef] [PubMed] 